\title{
Interactive Attendance System for Modern Education Using Computational Intelligence
}

\author{
V. Dhilip Kumar ${ }^{1}$ Md Meraj Alam, and Kemal Polat ${ }^{2, *}$ \\ ${ }^{1}$ Department of Computer Science and Engineering, Vel Tech Rangarajan Dr.Sagunthala R\&D Institute of Science and \\ Technology \\ ${ }^{2}$ Department of Electrical and Electronics Engineering, Faculty of Engineering, Bolu Abant Izzet Baysal University, \\ 14280, Bolu, Turkey, Email: kpolat@ibu.edu.tr \\ *Corresponding Author
}

How to cite this paper: V. Dhilip Kumar, Md Meraj Alam, and Kemal Polat (2021). Interactive Attendance System for Modern Education Using Computational Intelligence. Journal of the Institute of Electronics and Computer, 3 75-86. https://doi.org/10.33969/JIEC.2021.31006.

Received: August 9, 2021

Accepted: September 14, 2021

Published: September 23, 2021

Copyright (C) 2021 by author(s) and Institute of Electronics and Computer. This work is licensed under the Creative Commons Attribution International License (CC BY 4.0).

http://creativecommons.org/licenses/by/4.0/ (c) (i) Open Access

\begin{abstract}
The modern age is marked exclusively by advancements in digital technology. Modern education is not untouched by this advancement. However, there are few systems at present, which use computational intelligence in modern education. Currently, digital technology is bounded by commercial uses. In particular, face recognition is mainly used in criminal identification, security, payment, advertising, and healthcare. This project, Interactive Attendance System For Modern Education Using Computational Intelligence, is an attempt to combine modern education with modern computing. It uses the concept of Face Recognition to identify the person and to create an attendance file. The coding is done in the Python language on a Raspberry Pi 3B+ kit with Raspbian. Image pre-processing is used to convert captured images into grayscale and to extract areas of interest, i.e., faces. Machine learning is used to train the system to recognize faces using the LBPH Face Recognizer. This system can be installed in any modern educational institute for smart attendance.
\end{abstract}

\section{Keywords}

Face Recognition, Raspberry Pi 3B+, LBPH Face Recognizer, OpenCV, Attendance File

\section{Introduction}

Face recognition was first performed in 1964 by Bledsoe, Helen Chan, and Charles Bisson for an unnamed secret agency. Previously, different landmarks on the face, such as the eye centers, mouth, and so on, were manually marked and then supplied into a computer, which mathematically rotated them to correct for position fluctuation. To determine identity, the distances between landmarks were estimated by computer and compared between photographs. This first project was known as 
man-machine because a human effort was needed to extract features of the faces, such as the distance between the pupils of the eyes, the width of the mouth, etc. These features were normalized with the help of a computer for various factors, such as orientation, tilt, distance from the camera, lighting, etc. In 1966, the project was continued by Peter Hart at the Stanford Research Institute. Soon, computers will outperform humans at recognizing faces. Christoph von der Malsburg and graduate students from the University of Bochum in Germany and the University of Southern California in the United States created powerful facial recognition algorithms in 1997. Following these institutes, the Massachusetts Institute of Technology and the University of Maryland devised a system that functioned extremely effectively. The Face Recognition Grand Challenge (FRGC) was held in 2006, with the methods presented representing considerable improvements over earlier systems. Systems that surpassed humans in 3D face recognition and identifying identical twins were developed.

There is a great scope of this project in width as well as in-depth. The content of the project is listed below:

1) To recognize the face of students and professors.

2) To mark attendance along with time.

Heterogeneous face recognition (HFR), according to Decheng Liu et al. [1], is a difficult problem in face recognition due to considerable textural and spatial structure heterogeneity in face pictures. In reality, unlike traditional face recognition inhomogeneous situations, there are multiple face photos taken from various sources (such as different sensors or motors). We naturally use explicit invariant semantic information (facial at-tributes) to assist bridge the gap between multiple modalities, as motivated by human cognitive mechanisms. Deep neural network (DNN) architecture-based models have strong expressive power and learning capability, according to Gaurav Goswami et al. [2]. They are, however, effectively a black box method because mathematically formulating the functions acquired within their multiple layers of representation is difficult. As a result of this realisation, numerous academics have begun to develop strategies to exploit the flaws in deep learningbased algorithms, calling their robustness into doubt and exposing their singularities. Deep coupled framework to address the challenge of matching sketch picture to a gallery of mugshots, as reported by Seyed Mehdi et al [3]. Face drawings contain all of the necessary information about the spatial topology and geometric aspects of faces, but they lack critical facial characteristics like ethnicity, hair, eye, and skin color. In order to improve the sketch-photo identification performance, we propose a linked deep neural network architecture that makes use of facial features. A wide range of methodologies for 2D face recognition (FR) systems can be discovered in 
the literature, according to Guilherme F.P. et al. [4], due to its great applicability and challenges that need additional exploration, such as occlusion, differences in scale, facial expression, and illumination. A rising number of better 2D FR systems based on Swarm Intelligence and Evolutionary Computing algorithms have appeared in recent years. The current study provides an up-to-date Systematic Literature Review (SLR) on the application of Swarm Intelligence and Evolutionary Computation in 2D FR systems. Face is our primary focus of attention, and it plays a vital role in transmitting identity and emotions, according to Nijara Kalita et al. [5], who suggested a Survey on Face Recognition Based Security System and its Applications (2018). We encounter a large number of faces throughout our lives, but despite changes in visual input caused by ageing, beards, spectacles, or hairdo changes, it is quite easy to recognise them. Face Recognition is a technology that can recognise or verify a person based on a digital image or a video frame from a video source. Face identification is a difficult problem to solve in image analysis and computer vision. The goal of this research, according to Weidi Xie et al. [6], is to determine if two sets of photographs of a face are of the same person or not. The set-wise feature descriptor is traditionally calculated as an average of the descriptors from each individual face image in the set. We propose a neural network architecture that learns to aggregate based on both visual and content quality in this research. Battista Biggio et al [7]. Proposed a review of Learning-based pattern classifiers, including deep networks, have shown excellent performance in numerous application fields, spanning from computer vision to cybersecurity, ten years after the advent of adversarial machine learning (2018). However, it has been demonstrated that adversarial input perturbations, deliberately created either during training or during testing, can easily undermine its predictions.

Yuan Gao et al. [8] discuss the challenge of face recognition when there are few, if any, labeled samples of the face we want to recognize. Furthermore, these examples are frequently tainted by nuisance variables, both linear (i.e., additive nuisance variables such as poor lighting or the use of glasses) and non-linear (i.e., non-linear nuisance variables such as the use of spectacles) (i.e., non-additive pixel-wise nuisance variables such as expression changes). Because there are so few labelled examples, it's difficult to eliminate these distracting variables between the training and testing faces and achieve good recognition results.

Deep learning techniques, particularly convolutional networks, have quickly become the methodology of choice for interpreting medical images, according to Geert Litjens et al. [9]. This study analyses nearly 300 contributions to the field, the most of which were published during the last year, and discusses the major deep learning ideas relevant to medical picture analysis. We look at how deep learning is being used 
for image classification, object recognition, segmentation, registration, and other tasks, and present succinct summaries of studies for each application area. The open challenges and prospective research directions are discussed [9]. According to Lema tre, et al. [10], unbalanced-learn is an open-source python tool-box that aims to provide a wide range of approaches to deal with the problem of imbalanced datasets, which is common in machine learning and pattern recognition.

\section{Method}

The proposed system, Interactive Attendance System for Modern Education Using Computational Intelligence, is simple yet elegant. First of all, the system asks for the name/ID of the subject, then the color image of the subject is taken, and it is converted into a grayscale image. After this conversion, area of interest, i.e., the face, is extracted. This is pre-processing of the image. This image is saved under folder Database/ (Subject Name/ID) and images are numbered 1,2, 3, etc. Finally, fifty samples of each subject are taken.

These samples are used to extract characteristics of faces and to normalize them mathematically. Then these characteristics are used to train the system for face recognition using the LBPH Face Recognizer tool. The data thus obtained after training is stored in the file trainer.yml. When recognizing the face, the file trainer.yml is read and matched with the current subject to map the face with the available database. After mapping the face with the database, Name/ID is of the subject is displayed in real-time. Based on the face recognition, an attendance file is prepared and the unique name/ID of the subject is written on the file. The architecture of the project is shown in Figure 1. 


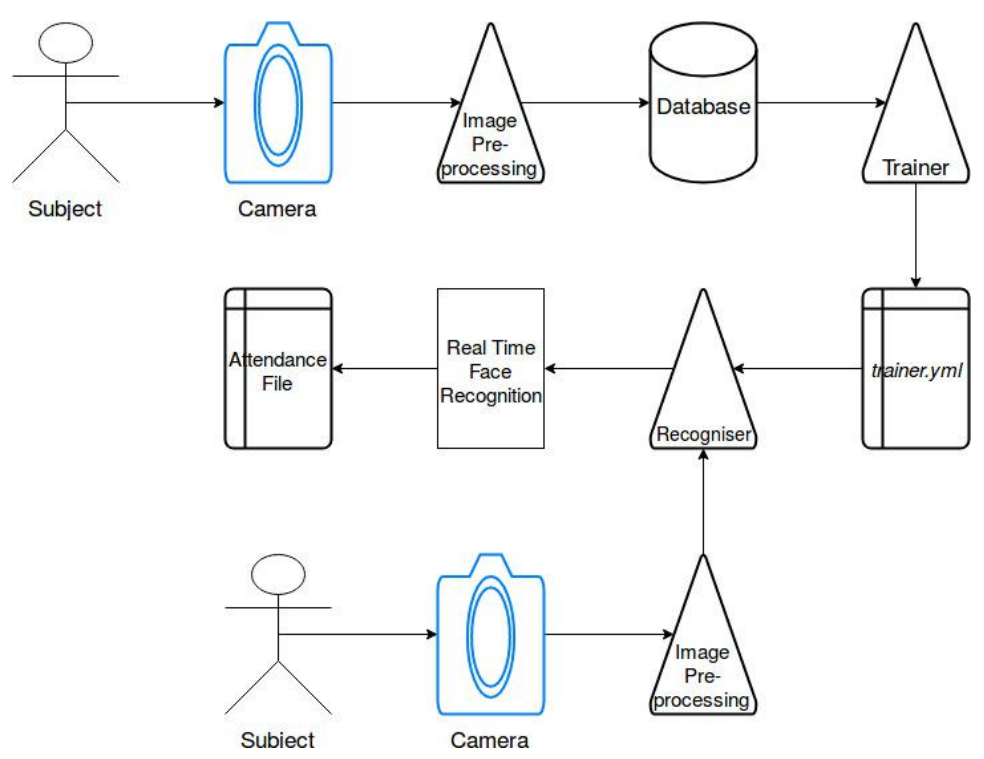

Figure 1. Architecture Diagram

\section{Algorithm:}

1. Database Creation

$$
\begin{aligned}
& \text { count }=0 \\
& \text { size }=0 \\
& \text { path = os:path } \\
& \text { video = cv2:VideoCapture(0) } \\
& \text { while(count }<50 \text { ) do: } \\
& \quad \text { (rval; image) = video:read() } \\
& \text { Image Preprocessing } \\
& \text { face = detectMultiscale } \\
& \text { if(face) then: }
\end{aligned}
$$

Resize Image

Write Image

count $=$ count +1

end if

end while

Destroy all windows

2. Datasets Training

Find Image in Database

Create list of Images; Labels; Names and Id

Create a numpy array 
model $=$ LBPHFaceRecognizer

Call model:train(a1; a2)

Call model:save(file)

3. Face Recognition and Attendance File

model $=$ LBPHFaceRecognizer

Call model:read(file)

count $=0$

size $=0$

path = os:path

video $=\mathrm{cv} 2$ :VideoCapture $(0)$

while(True) do:

(val; image) = video:read $($ )

Image Preprocessing

id; con = model:predict(a1)

if $($ con $<50)$ then:

Check id and assign name

Add name/id to a set list

else

Unknown

end if

end while

Create Attendance file

Write the set list in the file

Close Attendance file

Destroy all windows

\section{The Results and analysis}

To show the effectiveness of the real-time system image is taken and mapped. The face recognizer used in the project is LBPH Face Recognizer. The following formula can give its definition and recognition of face:

$$
\begin{aligned}
& \operatorname{LBP}\left(x_{c}, y_{c}\right)=\sum_{p=0}^{p-1} 2^{p} s(x)\left(i_{p}-i_{c}\right) \\
& \forall\left(x_{c}, y_{c}\right)=\text { centralpixelwithintensityi }{ }_{c} \\
& i_{p}=\text { intensityofneighboringpixel } \\
& \text { signfunction, } s(x)=\left\{\begin{array}{c}
1 ; \text { if } x \geq 0 \\
0 ; \text { else }
\end{array}\right.
\end{aligned}
$$

Euclidean distance is used to compare histograms and thereby to identify the person. 
The following formula gives Euclidean distance:

$$
\begin{aligned}
& e=\sqrt{\sum_{i=1}^{n}\left(\operatorname{hist}_{i}-h i s t 2_{i}\right)^{2}} \\
& \forall e=\text { euclediandistance } \\
& i=\text { instances } \\
& n=\text { totalnumberofinstances } \\
& \operatorname{hist}(j)=j^{\text {th }} h \text { histogram }
\end{aligned}
$$

Different steps followed in the project are:

Steps I: Noise in the image is filtered as follows:

1) Flip image matrix:- The image matrix is flipped so that the last column becomes the first column and vice-versa. Similarly, other columns are flipped.

2) Grayscale conversion:- The given RGB image is converted to Grayscale.

3) Resize image:- The grayscale image is resized keeping the aspect ratio.

Then save the images from forming a database, as shown in Figure 2.

Step II: Read the image file from the database. Then convert the image into numpy array using numpy function numpy:array(a). Training of database images is done as shown in Figure 3. And, finally, trained data is saved in the file 'trainer.yml'.

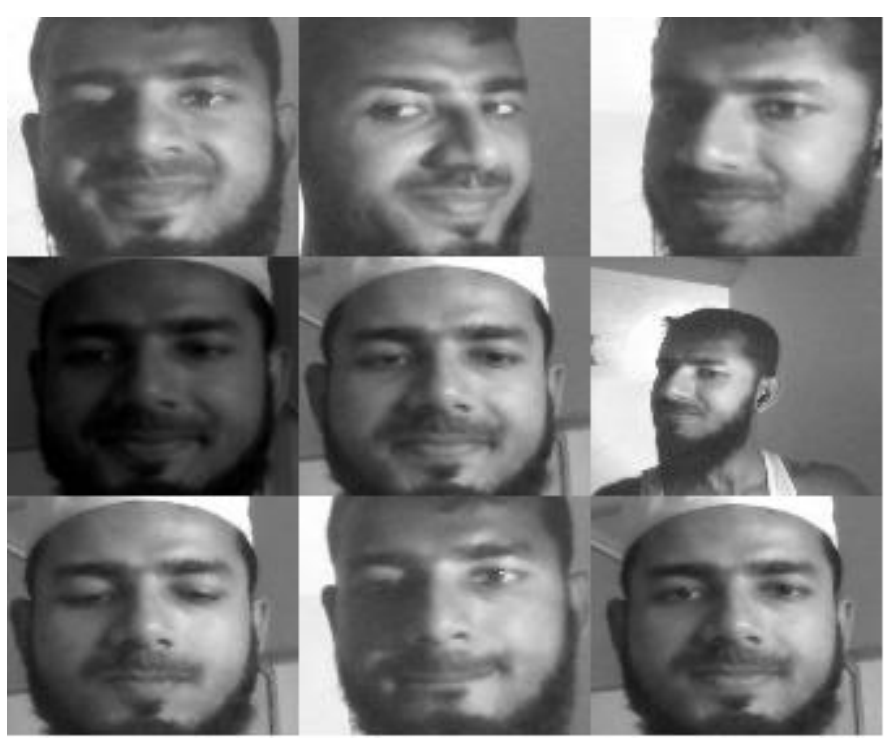

Figure 2. Database

Step III: Read trained data from the file 'trainer.yml'. Feed the system with the characteristics/ features of the face. Then take the image of the subject and preprocess it as given in Step I. Get Id and Confidence of the real time image and map it with the images saved in the database using 'trainer.yml'. Then display the name/id of the face recognized as shown in Figure 4. Then prepare Attendance file. 


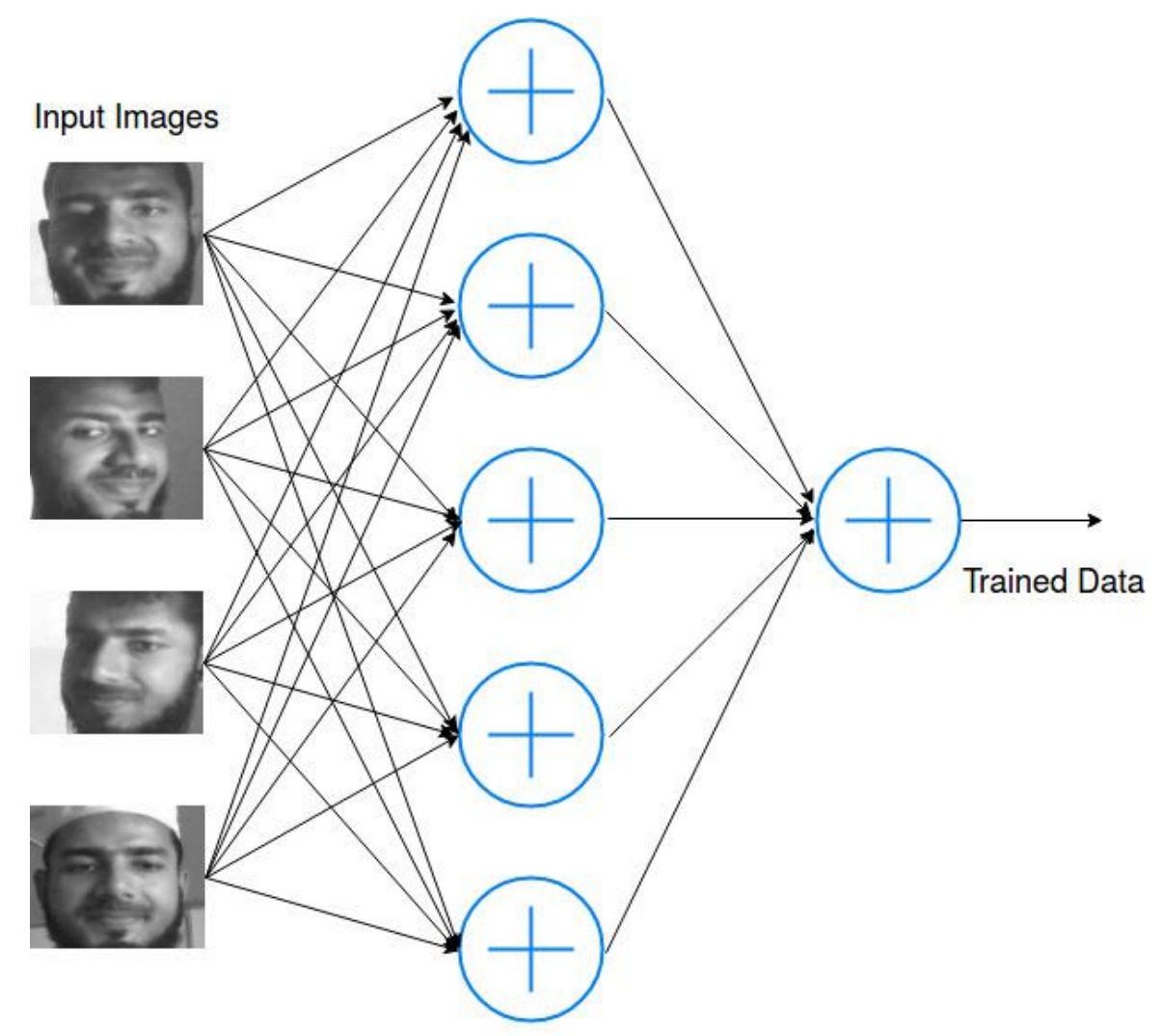

Hidden

Figure 3. Dataset Training

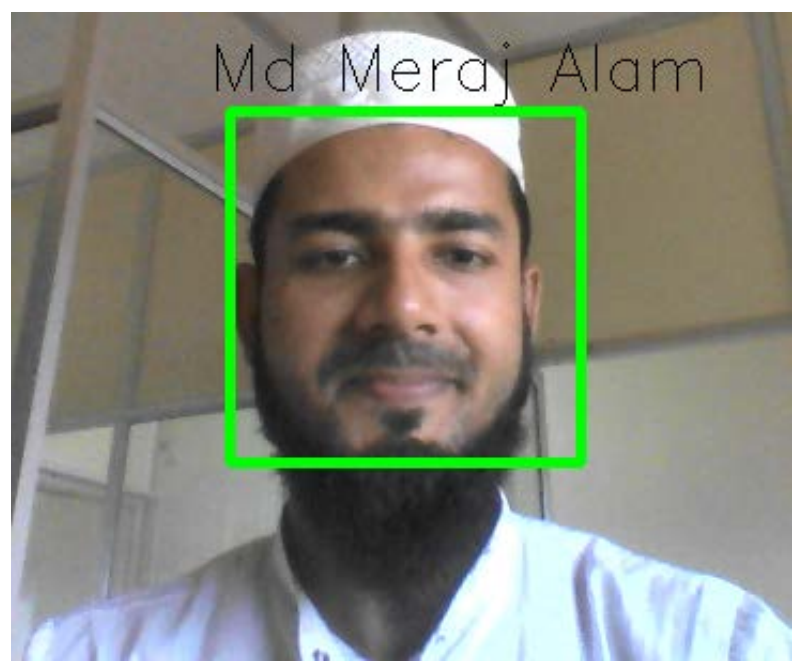

Figure 4. Result / Face Recognition 


\section{Discussion and Conclusions}

The traditional attendance system is time-consuming and, at times, proven ineffective. It has a high degree of chances of proxy attendance. This paper introduces an automatic attendance system with biometric face detection, thereby reducing the chances of proxy attendance. It takes less time as compared to other systems. The generated is in pdf form. Thus, it nullifies the chances of modifying the file.

The hardware for the project is shown in Figure 5. It shows camera, indicators, power adapter, HDMI port, Raspberry Pi 3B+, Laptop. 256-dimensional graph of one of the pictures in a database is shown in Figure 6. A histogram of the pixels of one of the samples in the database is shown in Figure 7. It shows that most of the pixels are shifted towards either white or black, which was expected since the images are saved in grayscale. Finally, a histogram of the confidence value is shown in Figure 8. It shows that the confidence value is highest and frequent in the range of 65 and 70 .

Future works include using face detection in a 3D model, improving database security, and uploading the file on a remote server using IoT.

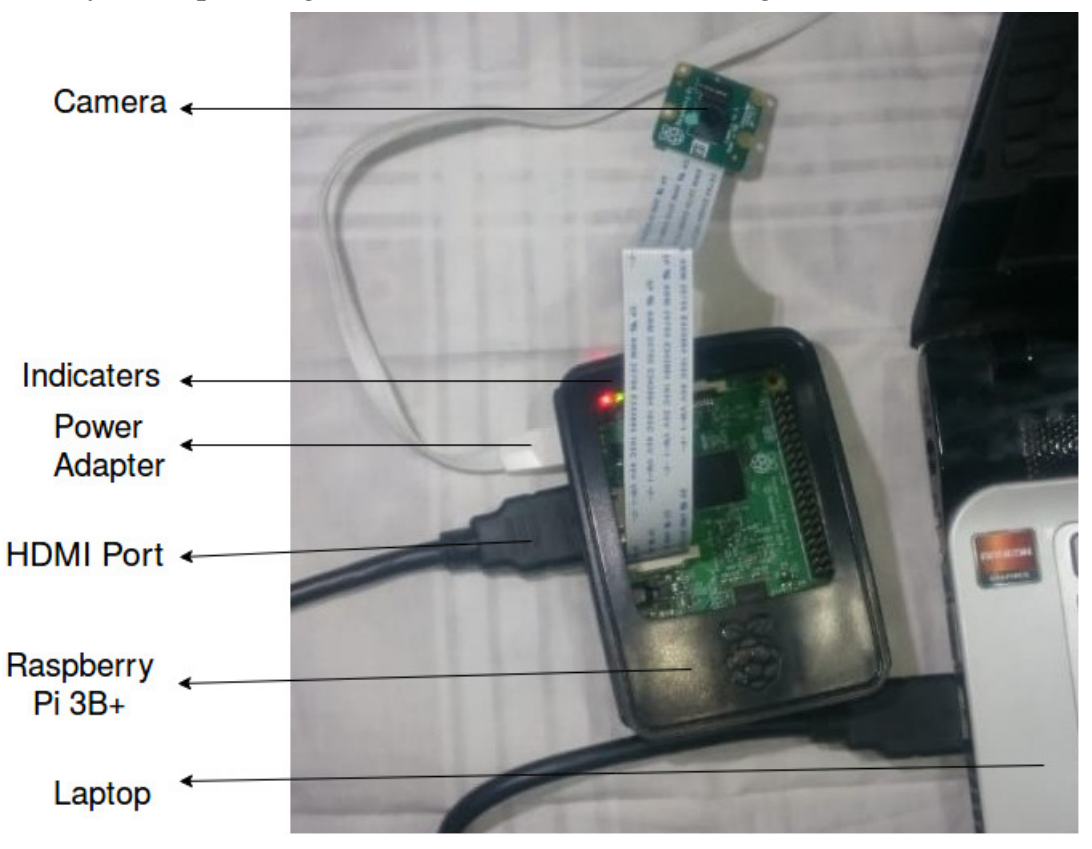

Figure 5. Hardware 


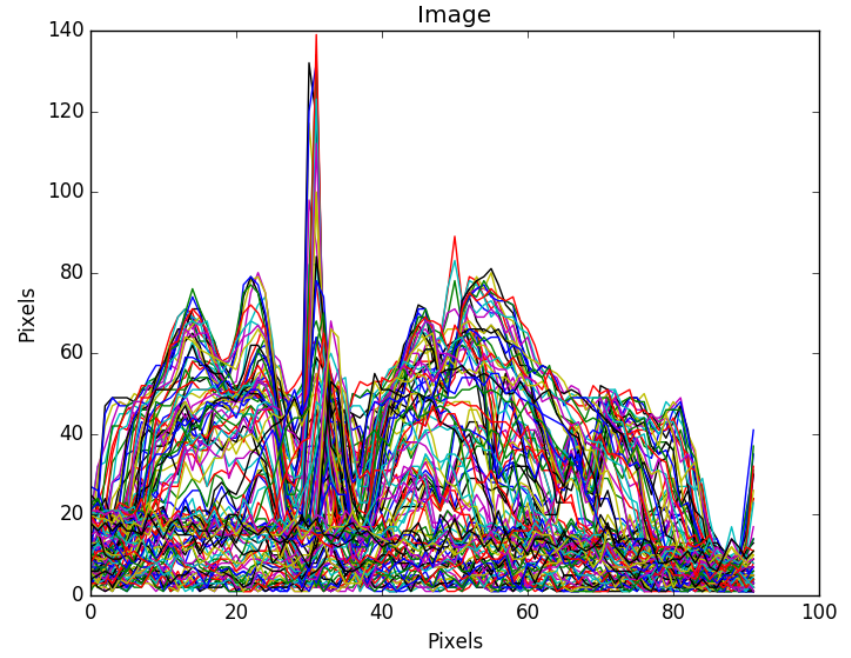

Figure 6. 256 D Image/Vector

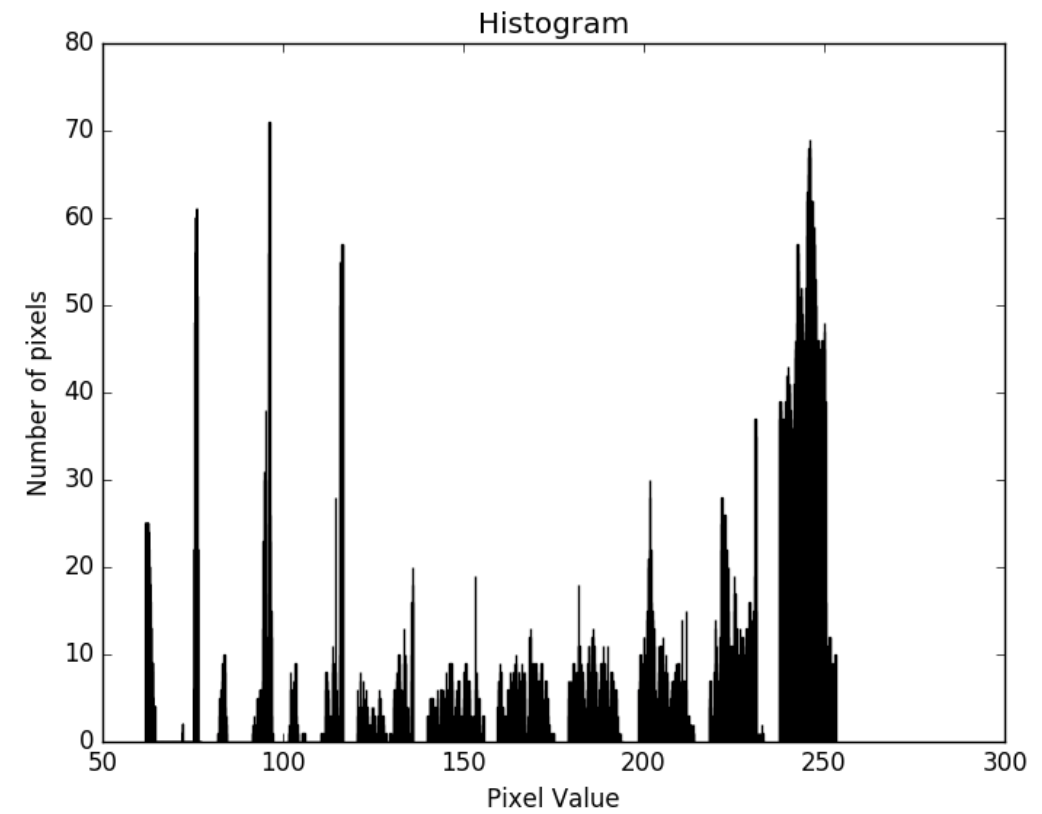

Figure 7. Histogram of pixels of the image 


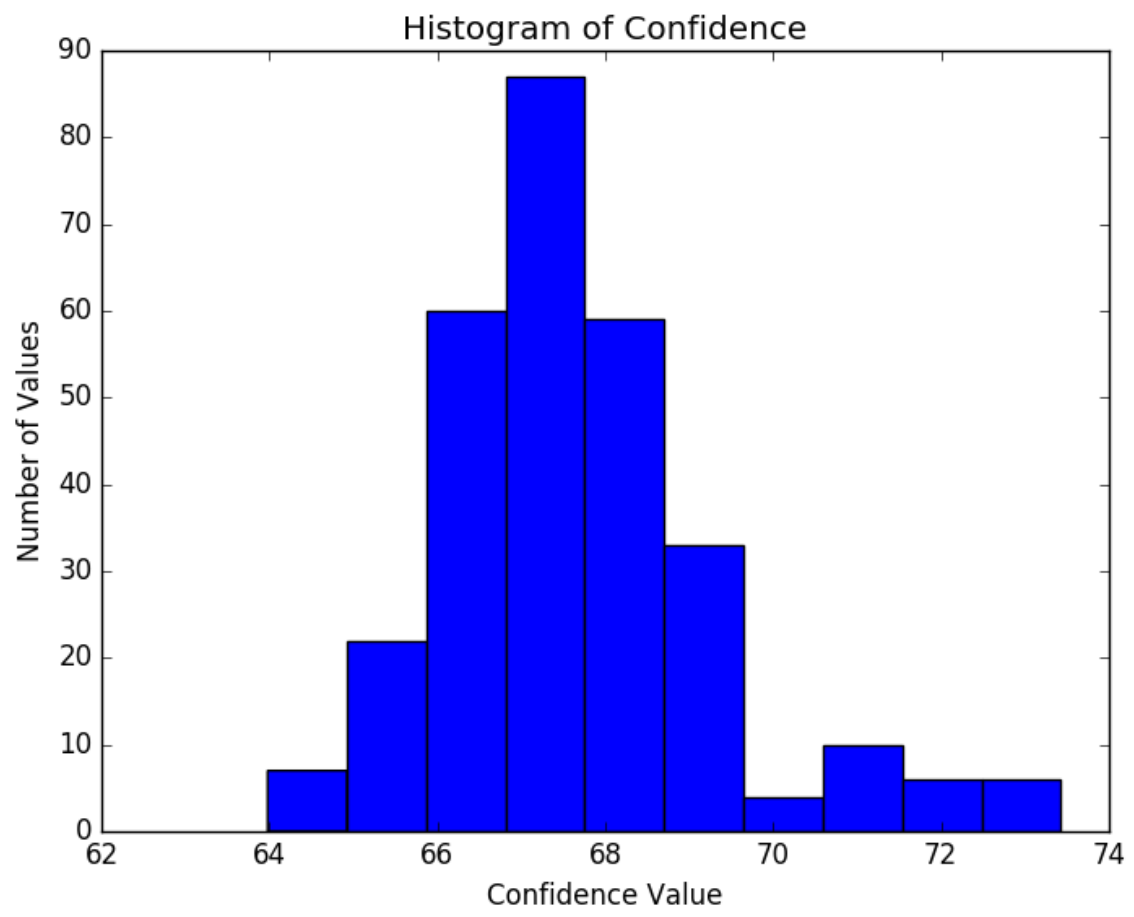

Figure 8. Histogram of confidence values

\section{Conflicts of Interest}

The authors declare that they have no conflict of interest.

\section{References}

[1] Deep Attribute Guided Representation for Heterogeneous Face Recognition, Liu, Decheng and Wang, Nan-nan and Peng, Chunlei and Li, Jie and Gao, Xinbo, http://www.ijcai.org/proceedings/2018/0116.pdf, 2018

[2] Unravelling Robustness of Deep Learning based Face Recognition Against Adversarial Attacks, Goswami, Gaurav and Ratha, Nalini and Agarwal, Akshay and Singh, Richa and Vatsa, Mayank, arXiv:1803.00401v1, 2018

[3] Deep Sketch-Photo Face Recognition Assisted by Facial Attributes, Iranmanesh, Seyed Mehdi and Kazemi, Hadi and Soleymani, Sobhan and Dabouei, Ali and Nasrabadi, Nasser M., http://arxiv.org/abs/1808.00059, 2018

[4] Swarm intelligence and evolutionary computation approaches for 2D face recognition: a systematic review, Plichoski, Guilherme Felippe and Chi-dambaram, Chidambaram and Parpinelli, Rafael Stubs, Revista Brasileira de Computac ao Aplicada, http://seer.upf.br/index.php/rbca/article/view/8046, 2018 (vol-10) [5] A Survey on Face Recognition Based Security System and its Applications, Kalita, Nijara and Saikia, International Research Journal of Engineering and Technology, 2018 (vol-5) 
[6] Multicolumn Networks for Face Recognition, Xie, Weidi and Zisserman, Andrew, http://arxiv.org/abs/1807.09192, 2018

[7] Wild patterns: Ten years after the rise of adversarial machine learning, Biggio, Battista and Roli, Fabio, Pattern Recognition, 2018 (vol-84)

[8] Semi-Supervised Sparse Representation Based Classification for Face Recognition with Insuficient Labeled Samples, Gao, Yuan and Ma, Jiayi and Yuille, Alan L., IEEE Transactions on Image Processing, 2017 (vol-26)

[9] A Survey on Deep Learning in Medical Image Analysis, Litjens, Geert and Kooi, Thijs and Be-jnordi, Babak Ehteshami and Setio, Arnaud Arindra Adiyoso and Ciompi, Francesco and Ghafoorian, Mohsen and van der Laak, Jeroen A. W. M. And van Ginneken, Bram and Sanchez, Clara I., http://arxiv.org/abs/1702.05747http://dx.doi.org/10.1016/j.media.2017.07.005, 2017 [10] Imbalanced-learn: A Python Toolbox to Tackle the Curse of Imbalanced Datasets in Machine Learning, Lema $\wedge$ tre, Guillaume and Nogueira, Fernando and Aridas, Christos K., The Journal of Machine Learning Re-search, http://www.jmlr.org/papers/volume18/16-365/16-

365.pdfhttps://dl.acm.org/citation.cfm, 2017 (vol-18) 